\title{
Task predictability and remembered duration
}

\author{
MARILYN G. BOLTZ \\ Haverford College, Haverford, Pennsylvania
}

\begin{abstract}
The effects of structural predictability on remembered duration judgments were examined within the context of the performance of a series of highly familiar tasks. Across a set of three experiments, task predictability was manipulated by the presence or absence of advance expectancies of what tasks were to be performed (Experiment 1), an (in)variant ordering of task performance (Experiment 2), and the placement of intermuptions at between- versus within-task locations (Experiment 3 ). In each case, a higher degree of predictability led to more accurate and reliable duration estimates that were relatively free of bias, while uncertainty decreased accuracy through an overestimation bias. These results not only render insight into the mediational mechanisms responsible for temporal judgments, but also suggest some practical applications for everyday behavior.
\end{abstract}

One of the most general goals of time-estimation research is to identify the factors contributing to the relative accuracy of duration judgments as well as certain systematic biases leading to over- or underestimations of an event's time span. This, in turn, not only provides insight into the temporal nature of everyday behavior, but, from a theoretical stance, suggests what underlying mediational mechanisms may be involved in the encoding and retrieval of event durations from the cognitive system.

According to several different reviews of the literature (Block, 1989; Hicks, Miller, \& Kinsbourne, 1976; Zakay, 1990), there are five general classes of variables that reliably influence temporal judgments: (1) the method used to assess duration estimates (e.g., reproduction, production, verbal estimation, comparative judgments); (2) characteristics of the experiencer such as age, gender, personality traits, degree of stress and arousal; (3) use of the prospective versus retrospective research design; (4) subjects' activity during a time span (e.g., cognitive processing demands of a task, active versus passive participation); and (5) characteristics of the events to be judged, including their total duration, sensory modality, and overall complexity.

Within this general categorization scheme, two factors are of particular theoretical interest because they are assumed to directly influence the cognitive mechanisms responsible for mediating duration judgments. The first of these involves the particular research design that is used and whether this is prospective or retrospective in nature. This distinction refers, in part, to the type of instructions administered to subjects in an experimental context, but, more importantly, is assumed to reflect different types of

This research was supported by a faculty research grant from Haverford College and was presented at the annual meeting of the Eastern Psychological Association in Philadelphia, March 1996. The author wishes to thank Tippony Scott for the collection of data. All correspondence should be addressed to M. Boltz, Department of Psychology, Haverford College, Haverford, PA 19041 (e-mail: mboltz@haverford.edu). cognitive processing activities. In prospective situations, subjects know in advance that a time judgment will be forthcoming, and so these estimates reflect the experience of time-in-passing. Of course, the drawback of this procedure is that subjects may engage in conscious timekeeping strategies (e.g., counting, tapping, monitoring an event's rhythm) that are artifactual to the experimental situation. Retrospective designs reduce, if not eliminate, this concern because subjects do not know a time judgment will be required until after an event has transpired. Given that estimates are assessed in an incidental fashion, they are assumed to reflect remembered duration. In reviewing the literature, it is clear that prospective designs have dominated much of the past research. To some extent, this is due to the influence of the psychophysical tradition and questions concerning the relationship between perceived and actual stimulus duration. In addition, unlike retrospective designs in which only one time judgment per subject can be obtained (before subjects become privy to the experiment's purpose), prospective situations are more economical, because they enable one to collect multiple estimates from a single subject. Nevertheless, interest in the study of remembered duration has recently increased. Retrospective designs allow one to investigate not only how certain variables influence the encoding and subsequent remembering of event durations, but also ways in which these may be linked to more general processing activities. This, in turn, has led to the development of several different theories of judged duration which all focus on a second variable of interest, namely, event complexity. As will be apparent in the following models, the notion of complexity has been defined in many different ways but in all cases is assumed to be the primary referent for duration judgments.

\section{Models of Remembered Duration \\ Memory-Based Models \\ One of the earliest approaches to adopt cognitive pro- cessing activities as a theoretical basis is a class of theo-}


ries known as the memory-based models. In general, they were developed to address relative duration judgments and a particular phenomenon known as the "filled interval effect." This refers to the finding that two equivalent time spans are not always judged as such-one interval is often estimated to be longer or shorter than the other. In an attempt to explain the underlying mechanisms responsible for this behavior, two different approaches have been offered.

The first is the storage-size hypothesis offered by Ornstein (1969). According to this view, more complex intervals will seem relatively longer than less complex ones, because the former consume more space, that is, more chunks of information, in memory. In support of this idea, Ornstein found that a randomized presentation of auditory sounds was judged relatively longer than the same sequence of sounds blocked by semantic category. Schiffman and Bobko (1974) reported a similar result with visual stimuli: lights that flashed in a random sequence were judged to be longer than those that flashed in a regular and rhythmic fashion. In both experiments, then, a haphazard stimulus array led to a longer duration estimate because it presumably consumed more space in memory.

An alternative model, offered by Block (1985), claims that judged duration does not depend on the number of chunks in memory but, instead, on the number of changes experienced by an individual. Hence, one interval may seem longer than another because it displays more changes in stimulus attributes, task demands, or the surrounding environmental context. This model has also been empirically supported. For example, Block and Reed (1978) found that the time span was estimated to be significantly longer when subjects were presented with a word list and asked to alternate between shallow and deep processing than when a single mode of processing (shallow or deep) was consistently maintained throughout the course of the experiment. Similarly, it has been observed that changes in the experimental context (i.e., room in which a given event was presented, the particular experimenter present) also lead to increased duration estimates (Block, 1982).

In sum, memory-based models differ in the presumed referent for duration judgments, namely, the number of chunks or changes in memory, but adhere to the common assumption that remembered duration will lengthen with increases in the amount of information in memory. Block (1990) has referred to this strategy as an "availability heuristic." Given that people do not directly encode an event's duration into memory, they rely on the availability of salient information to infer this estimate.

\section{Attention-Based Models}

In contrast to memory-based models, which primarily focus on relative duration judgments, a second class of theories has extended this perspective by addressing the overall accuracy in which time spans are both experienced and remembered. Although several individual mod- els fall within this category (Brown, 1985; Hicks et al., 1976; Thomas \& Cantor, 1978), all have applied the resource allocation theory of selective attention (Kahneman, 1973; Navon \& Gopher, 1979) to the study of time-estimation behavior. A model offered by Zakay (1989) is perhaps the most representative of this overall approach in that it not only incorporates the ideas of other theorists but integrates these with certain assumptions from the memorybased perspective.

The primary assumption is that when subjects are performing any given activity, such as a set of math problems, attention is split between the task's temporal and nontemporal information, namely, the task's total time span and, in this case, the set of mathematical calculations. Temporal information is presumably encoded via a cognitive timer while nontemporal information is processed by its own independent mechanism. Both compete for a limited pool of resources such that increased attention toward one dimension will decrease performance on the other. This, in turn, leads to the prediction that time-estimation behavior will vary under prospective and retrospective circumstances.

In prospective cases, where subjects know that a time judgment is forthcoming, more attention will be allocated toward the cognitive timer. This should result in highly accurate and reliable estimates of a task's total duration but relatively poor performance on nontemporal information. However, as task difficulty increases, more attention will be allotted to nontemporal information, such that duration judgments will become not only less accurate and more variable, but also shorter because less duration is encoded into the cognitive system. Retrospective designs, on the other hand, will primarily direct attentional resources toward the processing of nontemporal information, because there is no apparent reason to encode durational information into memory. Relative to prospective designs, then, remembered duration judgments should be less accurate and more variable. In addition, subjects may rely on the availability heuristic, as suggested by Block (1985) and Ornstein (1969). Given that event duration is not encoded into memory, they rely on the only source of available information to infer duration judgments, namely, the number of chunks or changes within the array of nontemporal information. Hence, as task complexity increases, judged duration will be increasingly overestimated.

This overall approach to time-estimation behavior is a very appealing one, because it not only is linked to a more general theory of attending but is also able to address a wide range of phenomena within the current literature. For example, when comparing the use of prospective and retrospective designs, the judged duration of tasks is, in fact, more accurate and reliable with the prospective method (see, e.g., Brown, 1985; Brown \& Stubbs, 1992; Miller, Hicks, \& Willette, 1978; Zakay, 1993). In addition, manipulations of complexity have been observed to exert a differential effect on experienced and remembered duration. Although the results of these studies have sometimes 
been conflicting in nature, meta-analyses of the literature have shown that across the majority of experiments, prospective judgments typically become shorter with increases in task difficulty, whereas retrospective judgments become longer (Block \& Zakay, 1997; Zakay \& Meran, 1988 , as described in Zakay, 1989). However, despite these contributions to the literature, this integrative model displays certain limitations in its generality which, in part, stem from the underlying philosophical assumptions about time.

The most important of these concerns retrospective judgments. In particular, it is assumed that an event's temporal duration is not encoded into memory under retrospective circumstances. Instead, people's attention is presumably focused on nontemporal information alone, such that event duration is later inferred from some aspect of this memorial representation via the availability heuristic (i.e., the number of chunks or changes it contains). Given that nontemporal information is presumably independent of and does not reflect temporal information, this means that people should be incapable of remembering an event's duration with any degree of accuracy. This, however, is not the case. In several recent studies, it has been found that if subjects are repeatedly exposed to sets of melodies or sounds from the natural environment and are later unexpectedly asked to recognize or reproduce their inherent durations, performance is highly accurate and reliable (Boltz, 1992, 1994, 1995). In other words, provided that sufficient learning experience has occurred, event duration can be incidentally encoded into the cognitive system even though subjects' attending was directed toward the array of nontemporal information during the encoding phase.

\section{Structural Remembering Model}

The latter phenomenon has been interpreted within a framework known as the structural remembering approach (Boltz, in press), which, in many ways, is directly compatible with resource allocation theory but places a greater emphasis on the role of the environment in the ability to remember event durations. The basic assumption is that events vary in their degree of structural coherence, which in turn influences attentional processes and the ability to encode an event's temporal information in conjunction with its nontemporal information.

Highly coherent events are ones that display a very lawful arrangement of structure and hence a high degree of internal predictability. Although this can arise from many different sources, one can be found at the level of event timing, where it can be shown that in many cases the arrays of temporal and nontemporal information directly coincide with one another. For example, a structural analysis of a speech utterance will show an accompanying rhythm in which temporal accents in the form of pauses coincide with grammatical phrase boundaries (Goldman-Eisler, 1972; Grosjean, Grosjean, \& Lane, 1979) and the pattern of stress coincides with high-content words (Martin, 1972). Music displays a very similar type of arrangement, as shown in Figure 1, which depicts the opening bars of "The Star Spangled Banner." Notice that temporal accents, arising from the relatively longer half notes, coincide with the words "see," "light," "hailed," and "gleaming," which correspond to melodic or grammatical phrase boundaries. These phrase boundaries are typically marked by special pitch intervals, namely members of the tonic triad chord (Berry, 1976; Cone, 1968; Piston, 1978; Todd, 1985), and represent the beginnings of new variations or returns to the melody's underlying theme. The result is that the melody's rhythm directly reinforces the nontemporal arrangement of pitch intervals. This type of coherent arrangement is quite typical of the auditory environment and is found not only in speech and music, but also in a wide variety of other ecological sounds (Boltz, 1992).

From a cognitive perspective, highly coherent events facilitate encoding and remembering in a number of different ways. First, and most importantly, given that the event's temporal and nontemporal information directly coincide and are highly redundant to one another, there is no need to split attending among the two structural dimensions. Instead, both should be encoded into memory with no extra effort such that attending to temporal or nontemporal information alone will result in the incidental encoding of the other dimension. Both should therefore be available in memory, particularly with greater learning experience where the inherent organization of the

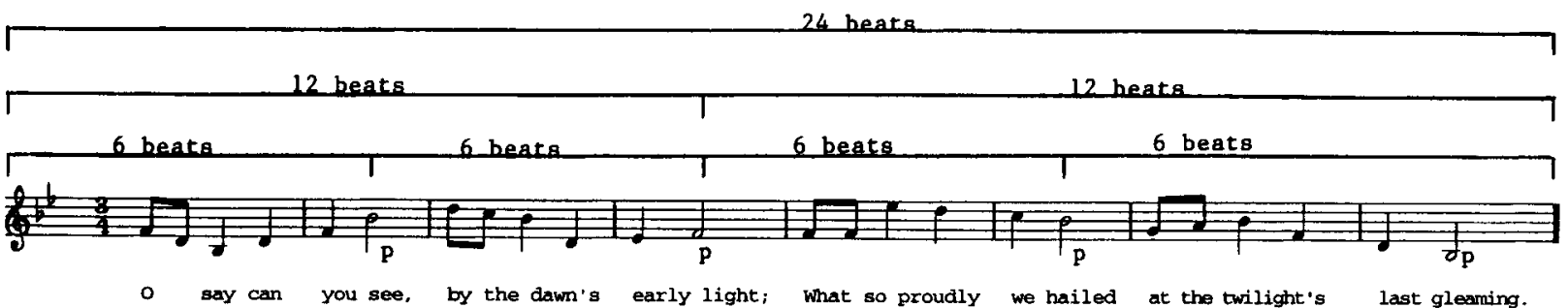

Figure 1. An example of a musical composition, "The Star Spangled Banner" by Francis Scott Key, displaying a highly redundant arrangement of temporal/nontemporal information. Within the melody's total time span, sequences of notes are nested within four melodic phrases (p) marked by members of the tonic triad chord (the tonic, dominant, tonic, and tonic, respectively). These phrase endings are attentionally highlighted by temporal accents $(d)$ and both regularly recur in time to outline higher order phrases. 
event will be more apparent. In addition, an event's timing should also facilitate encoding and remembering, because a regularly recurrent pattern of temporal accents not only allows one to easily attend to and track the event as it unfolds but also highlights the event's overall organization for learning. Similarly, just as accents can be used to guide attending, they also offer cognitive referents for recapitulating the event's structure from memory. These two factors working together, then-namely as a retrieval scheme in the form of rhythmic accents and as a direct redundancy between temporal and nontemporal information-lead to the prediction that the durations of highly coherent events will be remembered with a high degree of accuracy. This should be true in both prospective and retrospective situations in which the primary focus of attention on temporal or nontemporal information, respectively, should lead to an incidental encoding of the other dimension.

Although many naturalistic events are highly coherent, others are less so. This is perhaps most apparent during the early stages of learning. For example, the speech of a person learning a new, foreign language often displays pauses that fail to coincide with grammatical phrase boundaries. Similarly, the music of a novice pianist often shows temporal accents that conflict with melodic phrase endings and the inherent organization of pitch relationships. In both cases, the event's temporal and nontemporal information are structurally dissociated and independent of one another. Given that people are confronted with a seeming array of disjointed items that occur in an unpredictable fashion, these events, in addition to being difficult to track attentionally, will require separate processing resources for the encoding of both temporal and nontemporal information. This, in turn, leads to the prediction that an event's overall structure will also be poorly remembered, even with extensive learning experience. Although duration memory may be fairly accurate in prospective situations, this will not be the case in retrospective designs in which the primary focus is on nontemporal information. As suggested previously by the memory-based theorists (Block, 1985; Ornstein, 1969), people may resort to the availability heuristic and attempt to infer event duration from the number of chunks or changes within the array of nontemporal information.

Some support for this overall framework has been observed. In one set of studies (Boltz, 1992), subjects were asked to listen to a set of environmental sounds containing a single pitch value that rhythmically recurred in time and was thereby lawfully structured (e.g., sound of footsteps or panting), was continuous in duration (e.g., falling bomb or bowling ball rolling down an alley), or irregularly timed and thereby unpredictably structured (e.g., pecking of typewriter keys or sound of crying). After a varying number of learning trials, subjects were unexpectedly asked to reproduce or recognize the total duration of the sounds heard earlier. Results showed that while the durations of regularly timed and continuous events were well remembered, with a high degree of accuracy and low variabilty, the durations of less predictable events were poorly remembered regardless of the number of learning trials.

There is also evidence that coherent and incoherent events are encoded in a differential fashion. In Boltz (in press), subjects were presented with a set of musical compositions and asked to attend to either their temporal (i.e., total durations) or nontemporal information (i.e., sequence of pitch intervals) either alone or both in tandem. When the subjects were later unexpectedly asked to remember the inherent duration of each, the results showed that coherent melodies produced a high level of accuracy regardless of which structural dimension was attended to during the encoding phase. In addition, there was no decrement in performance when attention was split between the melodies' temporal and nontemporal information. Incoherent melodies, on the other hand, revealed evidence for independent processing resources. A high level of accuracy occurred only in the prospective group where subjects were attending to duration during the encoding phase. However, when attention was directed toward a melody's pitch or nontemporal information, duration accuracy declined, particularly when attentional demands increased due to the division of attention to both pitch and duration information. In these situations, people relied on the availability heuristic such that the tunes that contained more tonal segments were judged as being relatively longer.

As a set, then, these overall results extend the resource allocation theory of time-estimation behavior (Zakay, 1989) by illustrating that the ability to remember duration is dependent on event structure. Incoherent events, which primarily appear at the early stages of learning, display, at the level of event timing, structurally dissociated temporal and nontemporal information. As the resource allocation model predicts, the encoding of these events therefore requires independent processing resources such that duration accuracy occurs only when attentional resources are directed toward this dimension. Retrospective judgments, on the other hand, are highly inaccurate, because attending is primarily focused on nontemporal information alone. Given that relatively more effort is being expended on making sense of this information and the task at hand, few resources are left over to encode duration into the cognitive system. Hence, judgments are inferred from the salient aspects of the nontemporal array, which typically consist of the number of disconnected chunks or changes within memory.

The novel contribution of the structural remembering approach is addressing the time judgments of more predictable events. Again, these often emerge at later stages of learning where, on a perceptual level, it is apparent that the event's temporal information and nontemporal information directly coincide with one another and can therefore be jointly encoded into the cognitive system. This results in a high level of accuracy for both prospective and retrospective duration judgments that is often enhanced by an apparent rhythm that serves to guide both 
attending and retrieving. At a more general level, given that these events display a high degree of internal organization, fewer resources are needed to process and make sense of them, which in turn allows a greater allocation of resources toward the temporal dimension. In many ways, this concept is directly analogous to one within the more general cognition literature, where it has been found that tasks can become automaticized over time such that individuals can better divide their attention between two tasks at once (Posner \& Snyder, 1975). Here, it is suggested that a similar phenomenon occurs with the processing of information within an event.

\section{Generalizing the Predictability Concept}

The purpose of the present research was to extend the generality of these ideas by investigating some alternative ways in which structural predictability might manifest itself in the natural environment. To date, the concept of event coherence and predictability has primarily been considered at the level of event timing and the interrelationship between temporal and nontemporal information. Although it is clear how these constructs manifest themselves within the auditory environment, it is less apparent how they apply to the routine of everyday life. When one thinks about the types of activities normally performed during the course of a day, an analysis beyond the microlevel of event timing will reveal more global factors contributing to the overall predictability of an event. These can be attributed to a wide variety of sources, but in each case there is a lawful array of information that allows one to more easily attend to the ongoing course of the event and later to recapitulate it from memory.

One context that is particularly useful to investigate is requiring subjects to perform a set of tasks or activities over a given time span. This is highly representative of everyday behavior, because one's day, to a large extent, is defined by the sequence of actions that must be performed - for example, getting up in the morning, going to work, and doing the tasks required of one's job. Within this overall script, task predictability can arise in at least three different ways. The first, investigated in Experiment 1 , consists of simply knowing in advance what sequence of activities must be performed. For example, if a secretary is informed beforehand that (s)he must type an exam into the computer, schedule a series of speakers, enter some data into a spreadsheet, and order some supplies, (s)he knows exactly what (s)he is expected to do over the course of the morning. Assuming these tasks are well-learned ones that have been performed in the past, this individual can imagine or plan in advance what sequence of steps must be performed, how these should be done, and in what temporal order. This, in turn, grants greater predictability to the total time span than if (s)he is not instructed on what to do next until after a preceding task has been completed. The second source of predictability, investigated in Experiment 2, involved task ordering. If a set of required tasks is always performed in the same invariant order, this, too, grants greater predictability than if the ordering varies across successive occasions. Relative to the latter, which may appear as a series of disconnected chunks, an invariant ordering of tasks imposes a higher order organizational scheme, which should thereby facilitate both the encoding and the remembering of the event's total time span. Lastly, Experiment 3 investigated the occurrence of interruptions at between-versus within-task locations. Given that the former coincides with task boundaries, this should also yield greater accuracy in remembered durations.

\section{EXPERIMENT 1}

These ideas were investigated by asking advanced psychology students to perform a set of four familiar tasks that were routinely required within the psychology curriculum: word-processing or typing a text into a computer; proofreading a text for spelling errors; entering data into a spreadsheet; and calculating means on the computer for a series of data sets. The main manipulation in this first experiment was in the type of advance information subjects received. In one group, subjects were told ahead of time exactly what tasks they would be performing and the order in which they would occur. In contrast, a second group was not given any advance information about what they would be doing until immediately before a given task, that is, they did not know what the four tasks would be or the order in which they would occur until after the experiment was over. After completing the set of four tasks, subjects in both conditions were asked to verbally estimate, to the nearest half minute, the duration of each. If the construct of structural predictability is a generalized one that influences the ability to remember event durations, the same pattern of results as observed with manipulations of event timing should be obtained. Overall, the tasks that are more predictably structured should yield more accurate and reliable estimates than those that are less predictably structured. In addition, while the former condition should yield no systematic biases, the latter may give rise to systematic overestimations due to the seemingly disconnected nature of the tasks' time spans (i.e., availability heuristic).

\section{Method}

\section{Design and Subjects}

The design was a $2 \times 4 \times 4$ mixed factorial. Two groups of subjects who varied in the type of advance information they received (advance expectancies, no advance expectancies) were each asked to perform a set of four tasks over the course of the experiment (word processing-WP, proofreading-PR, data entry-DE, and the calculation of means-CM). The particular ordering of these tasks was counterbalanced across all subjects (I, II, III, IV).

Thirty-two students from the junior and senior psychology courses at Haverford College participated in the experiment in return for the opportunity to win three $\$ 10$ lottery prizes. Each had previously taken an introductory statistics course and a course in experimental psychology during the preceding $1 \frac{1}{2}$ years, in which they had routinely been required to analyze different data sets and report the results in APA-style manuscripts. 


\section{Stimulus Materials and Apparatus}

All four tasks were performed on a Macintosh Performa 6115CD computer. For the WP task, subjects were asked to type in a manuscript by Boltz (1991) that had previously been published in a psychological journal. This particular document was chosen simply because it was similar to the types of papers required in the department's curriculum. In the PR task, a second document, published by Boltz, Schulkind, and Kantra (1991), was presented on the computer and the spelling of one to three words on each successive line was altered. The third task required subjects to make a DE onto a spreadsheet that accompanied the Statistica software program (published by StatSoft, Inc., 2325 East 13th Street, Tulsa, OK 74104), a package used in all statistics courses within the department. These data were from a hypothetical study in which 100 males and $100 \mathrm{fe}-$ males were compared for differences in pain sensitivity as measured by the response latency to a hotplate. To enable subjects to enter these data into the computer, two columns on the spreadsheet were provided: the first for the codes of 1 and 2 to represent males and females, respectively, and the second for the dependent measure of response latency. Lastly, 60 raw data sets were prepared for the $\mathrm{CM}$ task, each consisting of eight four-digit numbers

\section{Procedure}

The subjects were individually tested and randomly assigned to one of four task orders (WP, PR, DE, CM; CM, DE, PR, WP; PR, WP, CM, DE; or DE, CM, WP, PR) and one of two instructional groups. They were told that the purpose of the experiment was to investigate people's ability to perform different types of cognitive tasks and that, since they would be using their hands, to remove all rings, watches, and bracelets so that these would not interfere with their motor performance.

Subjects in the first group (advance expectancies) were then informed of what would occur during the course of the experiment. They were given a brief description of the four tasks they would be performing and of the particular order in which these would occur. In contrast, subjects in the second group (no advance expectancies) were not given any such information; instead, they were simply told, immediately before it was presented, what task they would be performing. Hence, the complete set of tasks and their overall ordering was not known until the end of the experiment. At this point, the subjects began their participation in the four tasks. The instructions for each were as follows:

Word processing (WP). "The first task we'd like you to do is a word-processing one-that is, we'd like you to type this paper into the computer (where an open file for Microsoft Word was waiting). As you're entering this paper, please go at your own pace, the rate with which you are the most comfortable. Don't worry about proofreading what you've already entered or correcting any typographical errors. When I say START, please begin and continue to work until I say stop,"

Proofreading (PR). "In this next task, we'd like you to proofread a document that is displayed on the screen in front of you. We simply want you to carefully read through the paper and correct any errors you find. I should mention that all errors are simply spelling ones-the grammar and syntax should be fine and so don't worry about correcting these. When you see a spelling error, use the mouse to delete the word and then type in the correction. Again, please go at your own pace and work at the rate with which you are the most comfortable. When I say START, please begin and continue to work until I say STOP."

Data entry (DE). "Now we'd like you to enter some data into the spreadsheet shown on the screen in front of you. They represent data from a study in which males and females were assessed for any differences in pain sensitivity, as measured by how long they could keep their finger on a hotplate. All you need to do is make sure that the two columns of numbers you see on this piece of paper are entered into the appropriate columns of the spreadsheet. Again, please go at your own pace and work at the rate with which you are the most comfortable. Please begin when I say START and continue to work until I say STOP."

Calculation of means (CM). "In this last task, we'd like you to compute the mean of some data sets shown on the screen in front of you. The mean is determined by adding up all of the scores and then dividing by the number of scores. Please use the calculator on the Mac and enter the mean under its respective data set. As before, begin when I say START and continue to work at your own pace until I say STOP."

Each individual task was performed for $12 \mathrm{~min}$, a duration that was timed by the experimenter with a stopwatch. Following this, all subjects were unexpectedly asked to complete a questionnaire that assessed several different indices of behavior. They were first asked to estimate, to the nearest half minute, the duration of the entire experiment and how long it had taken them to complete each individual task. After this, they were asked to rate each of the four tasks, on a 7-point scale, for three different characteristics: its overall familiarity $(1=$ very familiar $)$, overall difficulty $(1=$ very easy $)$, and overall interest level $(1=$ very interesting $)$.

\section{Results}

To determine both the accuracy and bias of duration judgments, a set of ratio scores was determined for each subject that evaluated a task's estimated duration relative to its actual duration. Ratios equal to 1 represent perfect accuracy, those less than 1 indicate relative underestimations, and those greater than 1 indicate relative overestimations. One can also determine the mean percent error of subjects' estimates by subtracting a value of 1 from each observed ratio and then multiplying by 100 . For example, a ratio of 1.24 means that subjects, on average, overestimated a task's duration by $24 \%$. These mean ratio scores are shown in Figure 2 as a function of the two instructional conditions and each of the four tasks. Means have been collapsed over the counterbalancing variation of task order because this exerted negligible effects on performance $(F<1)$. In addition, the estimated duration of the experiment's total time span is not reported because, in all cases, this value was equal to the sum of the tasks' judged durations.

An overall analysis of variance indicates that the manipulation of advance expectancies exerts a significant influence on the accuracy and bias of duration judgments $\left[F(1,24)=7.29, M S_{\mathrm{e}}=.076, p<.001\right]$. As seen in Figure 2, the subjects who knew in advance what types of tasks would be performed and in what order yielded more accurate and reliable estimates than did the subjects who had no such information. In addition, while the former group shows no systematic bias (i.e., ratios equal to 1 ), the latter displays significant overestimations that, on average, are $38 \%$ longer than the tasks' actual durations. An interaction between task type and the instructional manipulation $\left[F(3,72)=4.61, M S_{\mathrm{e}}=.042, p<.01\right]$ further reveals that this overestimation bias is significantly higher in two of the tasks, namely, data entry and the calculation of means. An analysis of covariance indicated 


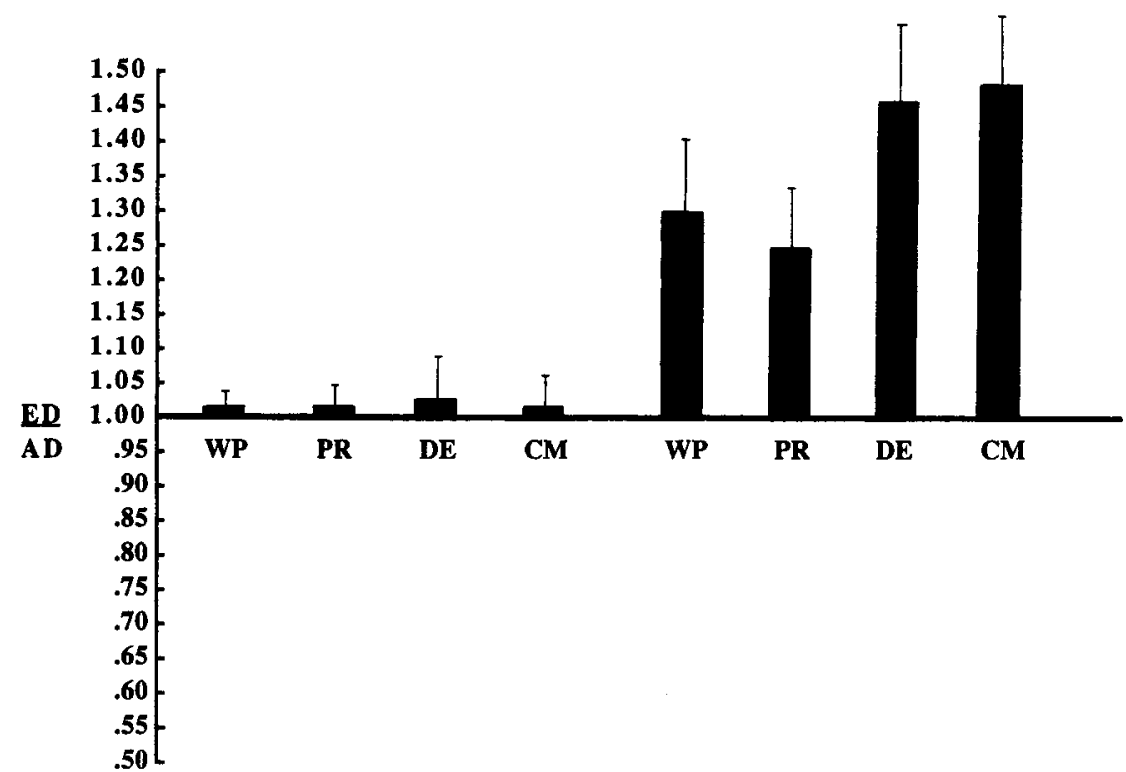

Advance Expectancies

No Advance Expectancies

Figure 2. Mean ratio scores (estimated duration/task's actual duration) in Experiment 1 as a function of the type of advance information subjects received and the set of tasks performed $(W P=$ word processing; $P R=$ proofreading; $D E=$ data entry; $C M=$ calculation of means). Ratios equal to 1 represent perfect accuracy; those greater or less than 1 indicate over- and underestimations of a task's duration, respectively.

that while all tasks are comparable in their degree of difficulty and interest, the tasks of data entry and the calculation of means are rated as less familiar to subjects $(\bar{x}=5.4$ and 5.8 , respectively) than are those of word processing $(\bar{x}=2.3)$ and proofreading $(\bar{x}=3.3)$ and significantly influence duration judgments.

Lastly, a final analysis was conducted to assess the percent frequency of subjects showing relative over- and underestimations in each condition of the experiment. These are shown in Table 1 . As previously suggested by the ratio data, subjects in the advance-expectancies condition are equally likely to make an over- or underestimation of each individual task. However, in the no-advanceexpectancies condition, the percent frequency of an overestimation is significantly higher than that of an un-

Table 1

Percent Frequency of Under-, Over-, and Accurate Estimations of Judged Duration in Experiment 1 as a Function of Task Type and the Instructional Manipulation of Advance Expectancies

\begin{tabular}{|c|c|c|c|}
\hline & Underestimations & Overestimations & $\begin{array}{c}\text { Accurate } \\
\text { Estimates }\end{array}$ \\
\hline \multicolumn{4}{|c|}{ Advance Expectancies } \\
\hline Word processing & 43.75 & 31.25 & 25.00 \\
\hline Proofreading & 50.00 & 31.25 & 18.75 \\
\hline Data entry & 37.50 & 43.75 & 18.75 \\
\hline Calculation of means & 43.75 & 43.75 & 12.50 \\
\hline \multicolumn{4}{|c|}{ No Advance Expectancies } \\
\hline Word processing & 31.25 & 62.50 & 6.25 \\
\hline Proofreading & 37.50 & 56.25 & 6.25 \\
\hline Data entry & 12.50 & 87.50 & 0.00 \\
\hline Calculation of means & 6.25 & 93.75 & 0.00 \\
\hline
\end{tabular}

derestimation in all task types, but particularly so in the data entry and the calculation of means tasks. These differences were confirmed by a chi-square analysis $\left(\chi^{2}=\right.$ $28.58, p<.01)$.

\section{Discussion}

The primary finding of this first experiment is that when people know ahead of time exactly what they will be doing during a given time span, this form of structural predictability leads to highly accurate estimates of the actual durations of tasks. Across all four tasks, the ratio scores of estimated-to-actual durations are indistinguishable from a value of 1 representing perfect accuracy. In contrast, the accuracy and reliability of estimates markedly decline when subjects have no advance information, and this is particularly true when they are performing relatively less familiar activities. In general, there are several factors that appear to be operating in tandem here to explain this overall pattern of results.

At a global level, advance expectancies allow people to plan ahead and to envision what set of steps will be required for each task and the overall temporal ordering of activities. This, in turn, grants a degree of integration to the total interval that is higher than it would be if one were not told what to do next until after a preceding task had been completed. In effect, the former condition enables people to conceive the span holistically, from beginning to end, and as it unfolds, the overall expected sequence of events matches one's preconception. One consequence is that fewer processing resources will be required, thereby making more resources available for the incidental en- 
coding of event duration. The second condition, on the other hand, creates a time span that is highly uncertain throughout because one has no idea what will happen next or when the final ending point will occur. Given that more processing effort will be needed here, duration is less likely to be incidentally encoded into the cognitive system, and so people will be more apt to rely on the availability heuristic to infer judged duration. In contrast to the more holistic nature of the time span in the advanceexpectancies condition, the changes from one task to another will be more salient and, as the memory theorists (Block, 1985; Ornstein, 1969) predict, this results in relatively longer time judgments. This is especially true with less familiar tasks where the sequence of required steps has not yet been integrated into a coordinated whole.

There is a second factor that is likely at play here, and it involves the mediation of expectancies. In a previous set of studies (Boltz, 1993), it has been found that when the actual amount of activity within a given time span (i.e., number of trials in a musical perception task) matches one's initial expectancies, retrospective duration judgments are highly accurate. In contrast, relative over- and underestimations arise, respectively, when the amount of activity is greater or less than expected. In addition, overestimations also arise in the absence of expectancies when one is waiting for an ending point that has not yet occurred and hence is late in coming. This overall scenario is directly analogous to the expectancy manipulation within the present experiment. In the advanceexpectancies condition, where the actual content and sequence of tasks conform to one's preconception, duration estimates are not only highly accurate and reliable but display no systematic bias. The no-expectancies condition, on the other hand, does yield an overestimation bias which, in turn, significantly decreases the overall accuracy of performance.

\section{EXPERIMENT 2}

Experiment 2 had three goals. The primary one was to examine a different form of predictability that could occur in the performance of a set of activities, namely task ordering. In particular, there are many jobs and vocations that require one to perform certain routines on a daily basis. On some occasions, these are done in the same invariant order; on other occasions, they are done in a varying order. For example, in the context of the present experiment, one may repeatedly perform the WP, PR, DE, and $\mathrm{CM}$ tasks in the same sequence over time or in a more random sequence. The question is, does this influence duration-judgment behavior?

From the perspective of the structural remembering approach, the higher degree of predictability afforded by invariant task ordering should influence cognitive processing activities in at least two ways. First, the repetitive performance of tasks creates a higher order rhythm, where the change from one task to another acts as a type of accent. Given that the individual tasks themselves always recur in the same sequence, one can anticipate in advance what the next task will be. This, in turn, will decrease the amount of attentional effort required and allow for more resources for the incidental encoding of event duration. In addition, the retrieval of this information should be facilitated by the overall rhythm that emerges, allowing people to reconstruct the interval from beginning to end. Overall, this means that duration estimates should be highly accurate and reliable and display no systematic bias. A randomized arrangement, on the other hand, will require more processing effort, because one does not know which task will occur next, and there is no overall rhythm to serve as a retrieval scheme during remembering. Hence, estimates will not only be less accurate but may reflect use of the availability heuristic in the form of an overestimation bias.

The latter prediction is related to the second purpose of this experiment, which was to assess effects due to the number of task segments on duration judgments. Although an increase in the amount of segmentation within an event has consistently revealed a corresponding increase in duration estimates (Poynter, 1983; Zakay, Tsal, Moses, \& Shahar, 1994), a recent set of studies by Boltz (1995) has shown that this effect depends on event structure. When the number of event segments is varied through manipulations in event timing, it is found that the segmentation bias is relatively absent in predictable events but is strongly apparent in unpredictable ones. The use of this availability heuristic, in fact, was assumed to contribute to the overestimation bias observed in the no-expectancies condition of Experiment 1. Here, however, it is examined more directly by varying the number of task segments. That is, in addition to having two groups of subjects who repetitively performed each task twice, in a predictable or unpredictable order, a third group was included who performed each task once, resulting in eight versus four task segments, respectively, across the different conditions. If effects from event timing generalize to the present context, then segmentation should exert a similar influence on the manipulation of task ordering. Relative to the four-segment condition, the variant ordering of eight task segments should produce longer estimations than would an invariant ordering.

A final goal was to compare time-estimation behavior under prospective versus retrospective circumstances. One assumption of the structural remembering approach is that while coherent events should yield comparable accuracy levels in both experienced and remembered duration, the duration estimates of less predictable events should be accurate only in prospective situations in which people can rely on conscious time-keeping strategies to aid performance. This pattern of results has previously been observed in the judged durations of coherent and incoherent melodies (Boltz, in press). If these findings also occur when different activities are being performed, then, in contrast to an invariant ordering of tasks, a varying order should yield different patterns of accuracy in prospective versus retrospective conditions. 


\section{Method}

\section{Design and Subjects}

The design was a $4 \times 3 \times 2 \times 4$ mixed factorial. All subjects performed the same set of tasks as in Experiment 1 (WP, PR, DE, $\mathrm{CM}$ ) but in one of three different arrangements (each task once; each task twice, predictable order; each task twice, unpredictable order). The two remaining between-subjects variables were type of instructions administered to subjects (prospective, retrospective) and counterbalance order (I, II, III, IV).

Ninety-six students from the junior and senior psychology courses at Haverford College participated in the experiment in return for the opportunity to win $10 \$ 10$ lottery prizes. Each satisfied the same criteria as in Experiment 1 and was randomly assigned to 1 of the 24 between-subjects conditions.

\section{Procedure}

Subjects in all conditions were tested individually. They were asked to remove all jewelry so that these would not interfere with the set of motor tasks they would be performing. They were then given a brief description of the four tasks and randomly assigned to one of three groups that varied in task ordering and the number of task segments. In the first condition, subjects performed each task once for $12 \mathrm{~min}$ before immediately going on to the next one. These tasks were arranged according to one of the four counterbalanced orders used in Experiment 1. Subjects in the second condition were asked to perform each task twice for $6 \mathrm{~min}$ each, with the ordering of the tasks being a predictable one. These too were performed within the four counterbalanced orders of Group 1 and then immediately again in the same sequence. The third condition was identical to the preceding one except that the tasks occurred in a more unpredictable order. That is, the subjects performed each of the four tasks within one of the four counterbalanced orders of Group 1 and then immediately again but in a different sequence. Across all three groups, the total duration of each of the four tasks was $12 \mathrm{~min}$, and although the subjects knew in advance what tasks would be required, they were not told in what order these would occur.

Before the beginning of the experiment, the subjects were also randomly assigned to one of two instructional groups. One was told that they would later be asked for a time judgment of each individual task (prospective); the upcoming time-estimation task was not mentioned to the second group (retrospective).

After the entire set of tasks had been completed, all subjects were asked to verbally estimate, to the nearest half minute, how much total time had been spent in performing each of the four tasks and, in addition, to rate each on a 7-point scale for its degree of familiarity, difficulty, and overall interest level.

\section{Results}

As in Experiment 1, a set of ratio scores was determined for each subject in order to evaluate a task's estimated duration relative to its actual duration of $12 \mathrm{~min}$. These mean values are shown in Figure 3 as a function of the four individual tasks, their overall arrangement, and the type of instructions administered to subjects.

An overall analysis of variance reveals a main effect for type of instructions $\left[F(1,72)=19.87, M S_{\mathrm{e}}=.0038\right.$, $p<.001]$, in which the subjects in the prospective condition produce more accurate and reliable estimates $(\bar{x}=$ $1.06)$ than those in the retrospective condition $(\bar{x}=1.16)$. This effect, however, is qualified by a significant interaction with task arrangement $\left[F(2,72)=11.5, M S_{\mathrm{e}}=\right.$ $.0038, p<.001]$. In the prospective condition, there are no differences between the three task arrays and, in all cases, the set of ratio scores does not significantly vary from a value of 1 , representing perfect accuracy. In effect, task ordering and segmentation exert no influence on behavior. The retrospective condition, on the other hand, produces a very different pattern of results. Here, the most accurate estimates are found when subjects perform each task twice in a predictable sequence, and once again the ratio scores are indistinguishable from a value of 1 . However, relative to this condition, estimates not only decline in their accuracy and reliability, but become significantly longer when subjects perform each task on a single occasion. In addition, this overestimation bias becomes even more pronounced when tasks are performed repetitively in an unpredictable order. These differences between the three task arrays were confirmed by a set of Tukey post hoc comparisons when $p$ was set to .05 .

These differential effects of task ordering/segmentation on prospective versus retrospective judgments were very robust ones that applied to each of the four tasks within the experiment. As in Experiment 1, the tasks were rated comparably on the dimensions of interest and difficulty but, in contrast to the word-processing and proofreading tasks, those of data entry and the calculation of means were considered relatively less familiar $(\bar{x}=1.9$ and 2.5 vs. 5.5 and 5.8, respectively). However, an analysis of covariance indicated that the overall pattern of duration estimates was independent of these perceptual dimensions.

Lastly, the percent frequencies of over- and underestimations were determined for each condition in order to assess the overall generality of results across all subjects within the experiment. These are shown in Table 2, where frequencies have been collapsed over the individual tasks. As can be seen, subjects in the prospective condition produced a relatively high frequency of accurate estimates, and when bias occurs, it is equally likely to be an over- or underestimation. In the retrospective condition, this same pattern of findings occurs when subjects performed each task twice in a predictable order. In contrast, the majority of subjects in the two remaining task arrays were significantly more likely to overestimate a task's duration $\left(\chi^{2}=14.16, p<.01\right)$. Again, these effects are reliable ones that apply to all four task types in each condition of the experiment.

\section{Discussion}

In contrast to Experiment 1 , the design of this second experiment enables one to more directly assess the underlying mechanisms assumed to mediate duration judgments. By manipulating the use of prospective versus retrospective instructions, this not only allows one to compare the encoding of event duration when time is consciously or only incidentally attended to, but to also examine whether task ordering and segmentation exert a differential influence on experienced versus remembered duration. And overall, results are consistent with the resource allocation theory of time-estimation behavior as 


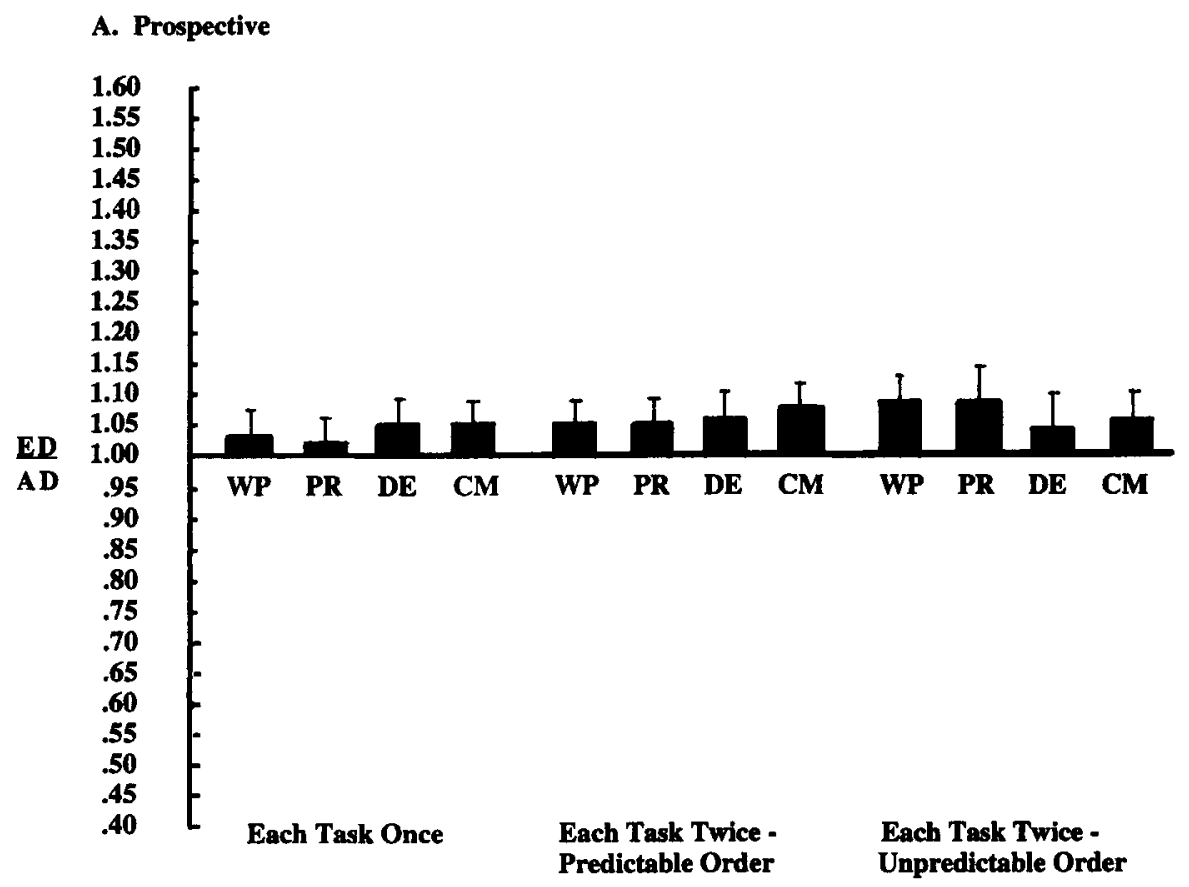

B. Retrospective

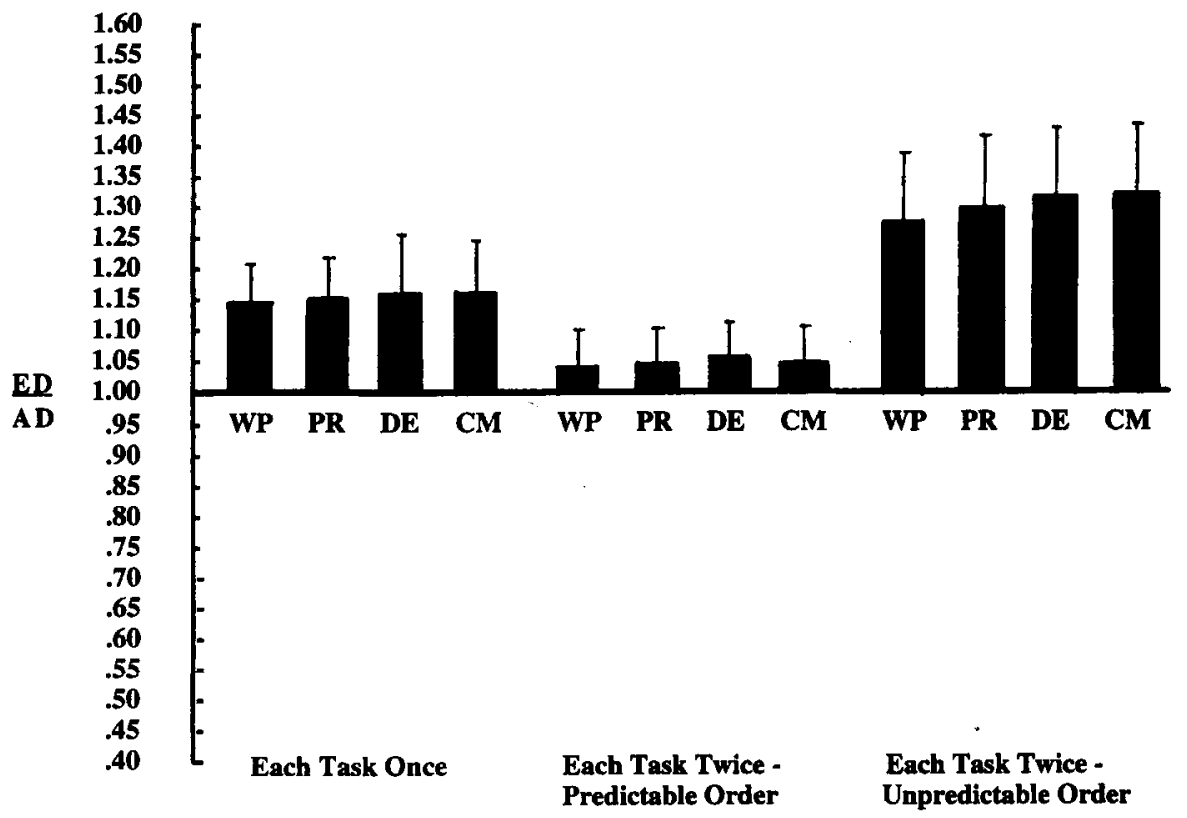

Figure 3. Mean ratio scores (estimated duration/task's actual duration) in Experiment 2 as a function of task type, task arrangement, and prospective versus retrospective instructions (WP = word processing; $\mathbf{P R}=$ proofreading; $\mathbf{D E}=$ data entry; $\mathbf{C M}=$ calculation of means). Ratios equal to 1 represent perfect accuracy; those greater or less than 1 indicate over- and underestimations of a task's duration, respectively.

it has been modified and extended by the structural remembering approach.

In the prospective condition of this experiment, when subjects were informed in advance that a time judgment would later be required, most attentional resources were directed toward the tasks' durations and the use of any time-keeping strategies to facilitate this encoding process. Hence, duration judgments were highly accurate and reliable, a finding that converges with several other studies in the past literature (Brown, 1985; Brown \& Stubbs, 1992; Miller et al., 1978; Zakay, 1993). In addition, task ordering and segmentation exerted no effects because 
Table 2

Percent Frequency of Under-, Over-, and Accurate Estimations of Judged Duration in Experiment 2 as a Function of Task Arrangement and Prospective Versus Retrospective Instructions

\begin{tabular}{lccc}
\hline & \multicolumn{3}{c}{ Uccurate } \\
& Underestimations & Overestimations & $\begin{array}{c}\text { Estimates } \\
\text { Prospective Judgments }\end{array}$ \\
$\begin{array}{l}\text { Each task once } \\
\begin{array}{l}\text { Each task twice: } \\
\text { predictable order }\end{array}\end{array}$ & 36.0 & 36.0 & 28.0 \\
$\begin{array}{l}\text { Each task twice: } \\
\text { unpredictable order }\end{array}$ & 34.0 & 31.0 & 34.0 \\
& Retrospective Judgments & \\
$\begin{array}{l}\text { Each task once } \\
\begin{array}{l}\text { Each task twice: } \\
\text { predictable order }\end{array}\end{array}$ & 14.0 & 37.5 & 25.0 \\
$\begin{array}{l}\text { Each task twice: } \\
\text { unpredictable order }\end{array}$ & 39.0 & 36.0 & 16.0 \\
\hline
\end{tabular}

these manipulations were essentially irrelevant to the goal at hand-namely, consciously monitoring time's passage. This finding is also consistent with past research by both Poynter (1983) and Zakay et al. (1994), who compared prospective and retrospective time judgments of word lists that had been segmented or unsegmented by high-priority events (i.e., Presidential names). In prospective situations, the nontemporal information of an event is presumably processed by its own independent mechanism, and such features as task ordering and segmentation are therefore ignored in temporal encoding activities.

In contrast, the retrospective judgments in this experiment were strongly influenced by manipulations of nontemporal information. Relative to the corresponding prospective conditions, the subjects who had performed each task once were much less accurate and showed an overestimation bias on the order of a $14 \%$ bias that became further enhanced $(29 \%)$ when subjects performed each task twice in an unpredictable order. Here, given the nature of the retrospective instructions, there was no need to attend to duration as the event transpired. In addition, although subjects knew in advance what the four tasks would be, their relative ordering was an arbitrary one that introduced uncertainty during the encoding phase and thereby increased the amount of processing effort. Hence, when estimating the task durations, subjects relied on the only source of information that was available in memory, namely, the number of task segments-the more segments present, the longer the time judgments.

The more interesting finding is the retrospective time judgments of tasks that had been performed twice in a predictable order. Overall, these events produced no systematic biases and yielded an accuracy level that was comparable to the prospective conditions. Given that the ordering of the second set of tasks matched the first, subjects could predict what the next task would be, which thereby freed more resources for the incidental encoding of event durations. In addition, the overall task arrange- ment in retrospect produced a higher order rhythm that could be used as a retrieval scheme for reconstructing durations of the tasks. Hence, even though more task segments were present here than in the condition in which each task was done once, the predictable array of segments led not to longer estimates as the memory and attention-based models would predict, but to shorter and more accurate estimates. This highlights the point that it is not merely the number of chunks and changes that matters in retrospective circumstances but the overall predictability of their arrangement.

As a set, then, the overall effects observed in this second experiment converge with previous manipulations of predictability introduced through variations in event timing and temporal accent structure (Boltz, 1995, in press). Although predictability can arise within many different levels of an event's structure, it nonetheless appears to exert a common influence on time-estimation behavior.

\section{EXPERIMENT 3}

This, final, experiment evaluated a different source of predictability which frequently occurs in everyday behavior. That is, when performing a set of tasks or activities over a given time span, one is often interrupted by various external factors - the ringing of the telephone or doorbell, conversations with others, and so on. Although any sort of interruption would appear to decrease the overall coherence of an event and disrupt cognitive processing activities, it may be that this depends on where the distractions occur.

Such a finding, for example, has previously been observed with the judged duration of musical compositions and filmed narratives (Boltz, 1995). In the latter, interruptions in the form of commercial breaks were placed at locations that either coincided (i.e., between episodes) or conflicted (within episodes) with grammatical phrase boundaries to yield coherent and incoherent events, respectively. When subjects were later unexpectedly asked to remember a film's duration, the results showed that the coherent versions yielded a higher level of accuracy than the incoherent ones. In addition, relative to a nocommercial condition, an increased number of commercials at episode boundaries significantly increased both the accuracy and reliability of judgments, while an opposite effect occurred when commercials occurred within episodes. Accuracy was not only lower in the latter condition but became increasingly longer as the number of commercials increased. These results suggest that, at a more global level, interruptions act as a set of accents that provide landmarks for remembering an event's time span. Hence, when these regularly recur and highlight the natural boundaries of an event's structure, they provide a higher order retrieval scheme that facilitates remembering. In contrast, interruptions that occur within natural boundaries detract attention away from the event's inherent structure, and the more interruptions 
there are, the more this organization is obscured. Estimates therefore become increasingly longer due to the number of seemingly disconnected chunks.

Experiment 3 was designed to determine whether a similar pattern of effects would emerge when a set of tasks were being performed. Two conditions from Experiment 2 were used, with subjects performing each of the four tasks (WP, PR, DE, and CM) twice in a predictable or unpredictable order. During the course of this, interruptions occurred in the form of phone calls to the experimenter, who was sitting in the same room as the subject. The key manipulation was where these interruptions occurred. In one condition, they always occurred between two individual tasks; in a second, they occurred within a given task. Two other conditions, in which no interruptions occurred, were included as controls. Afterwards, all subjects were unexpectedly asked to estimate each task's total duration. Given the results of Experiment 2, in which variations in predictability exerted an influence on remembered duration alone, all judgments in this third experiment were retrospective in nature.

If these types of interruptions form an overall accent structure, the results for the predictable task ordering should be analogous to those of the film study described earlier: relative to the no-interruption condition, phone calls occurring between tasks should yield a higher level of duration accuracy than should calls occurring within tasks. Predictions for the conditions displaying an unpredictable task order are somewhat less straightforward. Although the no-interruption group should show a low level of accuracy in the form of an overestimation bias, as in Experiment 2, these effects should increase in magnitude when interruptions occur within a given task. Distractions will provide an even greater degree of unpredictability and, given the increased number of task changes and segments, the tasks' durations should seem relatively longer in retrospect. However, the effects of placing interruptions between tasks are less clear. On the one hand, performance may be comparably poor due to the mere presence of distractions within a random ordering of tasks. Conversely, duration accuracy may actually improve due to the regular recurrence of interruptions. If these act as accents in memory, then the tasks that immediately follow may be attentionally highlighted and hence be better remembered. In essence, then, the interruption manipulation should exert a similar impact on both arrangements of task order.

\section{Method}

\section{Design and Subjects}

The design was a $4 \times 2 \times 3 \times 4$ mixed factorial where the sole within-subjects factor was the set of four tasks that all subjects performed. These tasks recurred in a predictable or unpredictable order and displayed one of three types of interruptions (between tasks, within tasks, none). Counterbalance order (I, II, III, IV) was the remaining between-subjects factor.

Ninety-six subjects participated in the experiment and satisfied the same criteria as in Experiment 2.

\section{Procedure}

The procedure was identical to the two conditions of Experiment 2 , where subjects performed each of the four tasks twice, in a predictable or unpredictable order, for a total duration of $12 \mathrm{~min}$ for each task. However, in addition to these two conditions, which served as controls, four other conditions, which varied in the placement of interruptions, were included. These interruptions were in the form of four brief phone calls to the room where the experiment was being conducted; in each case, the experimenter answered the phone and said, "No, (s)he isn't here right now," and then hung up. In two of the conditions, these interruptions always occurred immediately after the instructions had been presented to a subject and before the onset of the task itself. These occurred before the first, third, fifth, and seventh tasks, respectively, of the predictable and unpredictable task sequences. In the two remaining conditions, the interruptions were identical but occurred during the first, third, fifth, and seventh tasks. Within these four tasks, the phone calls occurred during the 4th, 2nd, 3rd, and 5th minute, respectively, of the 6-min task duration.

At the end of the experimental session, all subjects were unexpectedly asked to estimate, to the nearest half minute, the total duration of each of the four tasks and to also rate each for its degree of familiarity, difficulty, and overall interest level.

\section{Results}

The mean ratio scores of estimated to actual duration are presented in Figure 4 as a function of task ordering, task type, and placement of the interruptions. As before, means have been collapsed over the counterbalancing variation of task order, since this exerted negligible effects on performance $(F<1)$.

The overall analysis of variance reveals a main effect for task order in which a predictable sequence leads to greater duration accuracy than does an unpredictable one $\left[F(1,72)=31.83, M S_{\mathrm{e}}=.0049, p<.001\right]$. The more interesting finding, however, is a significant interaction between task order and the placement of interruptions $\left[F(2,72)=9.89, M S_{\mathrm{e}}=.0049, p<.001\right]$. As seen in the predictable-order conditions of Figure 4 (Panel A), duration estimates are very accurate and do not vary from a value of 1 when there are no interruptions within an experimental session. Relative to this control group, estimates are equally accurate when interruptions occur between tasks. However, the placement of interruptions within individual tasks produces a significant decrease in accuracy and reliability that is due to an overestimation bias. The unpredictable-task orders shown in Panel B reveal a somewhat different pattern of results. In the control group, there is a relatively poor level of accuracy in which overestimations occur on the order of $27.5 \%$. However, while this error rate and overestimation bias significantly increase to $36.5 \%$ when interruptions occur within tasks, it decreases by $10 \%$ when these same interruptions occur between tasks. These overall differences in performance are confirmed by a set of Tukey post hoc comparisons $(p<.05)$.

A secondary set of analyses was conducted to determine whether interruptions merely influenced the judged durations of the tasks with which they coincided (i.e., the first, third, fifth, and seventh tasks of each sequence) or exerted an impact on all tasks within an experimental session. The latter was determined to be true, and this ap- 
A. Predictable Task Order

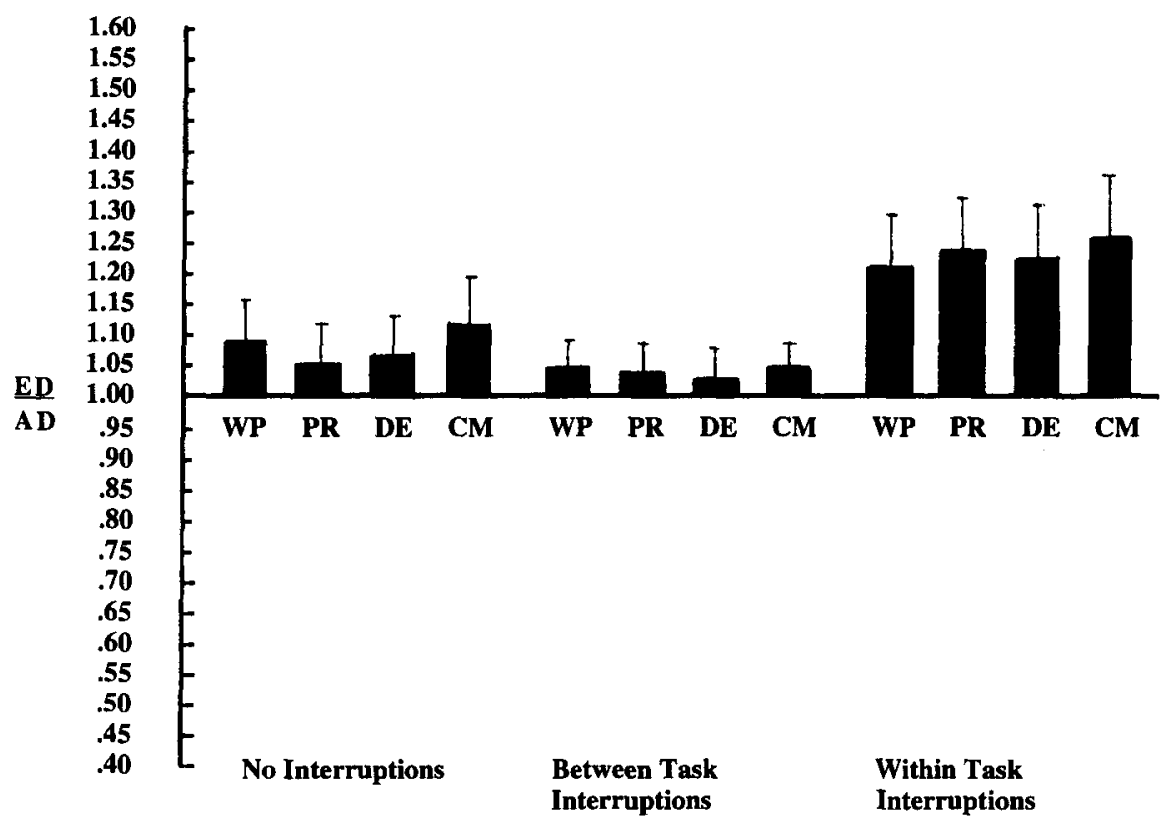

B. Unpredictable Task Order

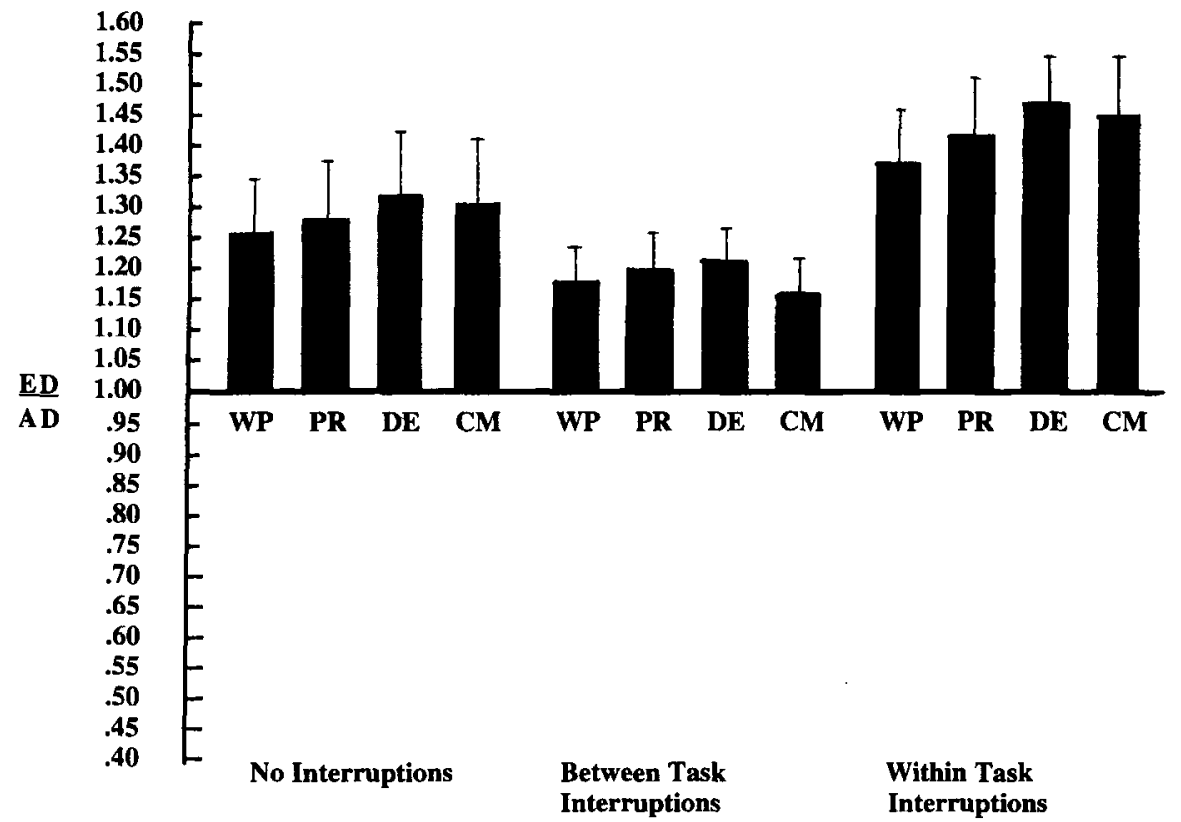

Figure 4. Mean ratio scores (estimated duration/task's actual duration) in Experiment 3 as a function of task arrangement, task type, and placement of interruptions (WP, word processing; PR, proofreading; DE, data entry; CM, calculation of means). Ratios equal to 1 represent perfect accuracy; those greater or less than 1 indicate over- and underestimations of a task's duration, respectively.

plied to the placement of interruptions both between and within tasks, as well as to both predictable and unpredictable task orders.

As a set, then, these overall results are fairly robust ones that generalize across different tasks. An analysis of covariance also reveals that they are not influenced by a task's degree of interest, difficulty, or familiarity. Lastly, an analysis of the percent frequency of over- and underestimations in each condition indicates that the pattern of bias is one that applies to the majority of subjects in the experiment. As seen in Table 3 , the conditions producing a high degree of accuracy display no systematic bias, 
Table 3

Percent Frequency of Under-, Over-, and Accurate Estimations of Judged Duration in Experiment 3 as a Function of Task Arrangement and Placement of Interruptions

\begin{tabular}{lccc}
\hline & Underestimations & Overestimations & $\begin{array}{c}\text { Accurate } \\
\text { Estimates }\end{array}$ \\
\hline & Predictable Task Order & \\
$\begin{array}{l}\text { No interruptions } \\
\text { Between-task } \\
\text { interruptions }\end{array}$ & 41.0 & 34.0 & 25.0 \\
$\begin{array}{l}\text { Within-task } \\
\text { interruptions }\end{array}$ & 37.5 & 34.5 & 28.0 \\
& 25.0 & 56.0 & 19.0 \\
$\begin{array}{l}\text { No interruptions } \\
\text { Between-task } \\
\text { interruptions }\end{array}$ & 12.5 & 79.5 & 8.0 \\
$\begin{array}{l}\text { Within-task } \\
\text { interruptions }\end{array}$ & 25.0 & 62.5 & 12.5 \\
\hline
\end{tabular}

while those yielding a low level of accuracy are accompanied by a high percentage of overestimations $\left(\chi^{2}=\right.$ $15.55, p<.01$ ).

\section{Discussion}

The results of this third experiment provide further evidence that the inherent structure of a time interval influences the overall accuracy in which it is remembered. Although external distractions can exert a deleterious effect on remembering, those that coincide with the natural boundaries of an event significantly improve the retrieval of the event's total time span. This has been observed previously with the duration memory of musical compositions and filmed narratives (Boltz, 1995) and was found here with task performance.

In the tasks that were predictably ordered, performance was indistinguishable from perfect accuracy when there were no interruptions during the experimental session. Given this ceiling effect, it was not possible to determine whether performance benefited from the placement of interruptions between tasks. However, the comparable conditions in the unpredictable-task sequences revealed a $10 \%$ improvement in accuracy and bias. At a higher order level, interruptions at these locations can act as cognitive referents that attentionally highlight the corresponding tasks and thereby allow one to more easily reconstruct the interval from memory. Although performance is still impaired from the arbitrary ordering of tasks, the regularly recurrent pattern of interruptions provides a higher order retrieval scheme that facilitates remembering.

In contrast, the occurrence of interruptions within tasks gives rise to an increase in error and an overestimation bias. Regardless of the overall ordering of the tasks, auditory distractions at these locations disrupt the ongoing flow of activity and direct attention away from the task itself. In addition to influencing the encoding of temporal information, an overall pattern of segmentation is imposed that conflicts with task boundaries and thereby de- creases the overall organization of the interval. The end result is a series of seemingly unrelated chunks and changes that, given their salience, lead to an overestimation bias via the availability heuristic.

\section{GENERAL DISCUSSION}

The results of the three experiments described here illustrate that the construct of predictability is a key factor in determining both the accuracy and bias of timeestimation behavior. In each case, a higher degree of predictability leads to more accurate and reliable duration estimates that are relatively free of bias, while uncertainty decreases accuracy through an overestimation bias. These overall results not only render insight into those mediational mechanisms responsible for temporal behavior, but also suggest some practical applications for everyday behavior.

Predictability can arise from many different aspects of an event's structure, but in each case grants greater coherence to a given time span. At the microlevel of event timing, temporal accents arising from pauses or prolonged durations can be demonstrated to bear lawful or unlawful ratio relations to an event's hierarchical arrangement of nontemporal information (Boltz \& Jones, 1986). This hierarchy is typically generated from an underlying grammar, such as that found within musical compositions (Lerdahl \& Jackendoff, 1983), speech utterances (Grosjean et al., 1979) and conversations (Jaffe \& Feldstein, 1970), narrative stories (Mandler \& Johnson, 1977; Thorndyke, 1977), or action movements (Newtson, 1973; Pierson, 1976). In any case, temporal accents either reinforce or conflict with phrase boundaries to attentionally highlight or obscure the event's inherent organization. Within the routine of everyday life, predictability can also arise from more global factors. Some of these were investigated here and include the extent to which an individual knows or does not know in advance what exactly will be required during a given interval, an invariant or variant ordering of activities, and the relative placement of interruptions during an ongoing task. Although it was not directly addressed here, learning and level of expertise can also contribute to the overall predictability of an activity by determining how well an individual knows what steps will be required and how these should be executed. This factor was held constant within the present set of studies in order to maintain methodological rigor, but it is, nevertheless, an important one, because the same event or activity will seem less coherent at early stages of learning than it will at later stages (Boltz, 1996).

Despite these different origins, predictability provides the common function of integrating an event and its total duration into an organized whole in which nested sets of subelements appear to be interrelated to one another. In addition, one can often generate expectancies about the event's ongoing course that are subsequently confirmed. In contrast, when predictability is absent, there is no overarching sense of organization; instead, the event ap- 
pears to consist of a number of segments or changes from one element to another that are all unrelated and disconnected from one another. From a theoretical perspective, this exerts a differential impact on cognitive processing activities, which in turn influence time-estimation behavior. Although many of these processes have been previously addressed by the memory- and attention-based models, others have been relatively unacknowledged but are nonetheless consistent with these approaches.

In the past, it has traditionally been assumed by both the attention- and memory-based models that the effects of prospective versus retrospective circumstances apply to all events, regardless of their inherent structure. When people know in advance that a time judgment will be required, attentional resources can be devoted to time-keeping strategies such that estimates will later be quite accurate. This, in fact, was found in Experiment 2. However, in retrospective situations, people are presumably incapable of encoding event durations with any degree of accuracy because attention is directed exclusively toward nontemporal information alone. To infer an event's total time span, people therefore rely on salient features of the nontemporal array, such as the number of chunks and changes, and the more features present, the longer the duration estimate. Although these assumptions were also supported in the present set of experiments, they applied only to less predictable events. In contrast to the models' predictions, highly predictable events were judged in retrospect at an accuracy level that was indistinguishable from the events' actual durations and displayed no systematic bias. Given their high degree of internal coherence, these events require fewer processing resources that enable one to incidentally encode duration into memory. In addition, there is often a higher order retrieval scheme within an event's structure itself that facilitates both encoding and remembering. At the level of event timing, remembering can be guided by temporal accent structure and, within the context of performing routine activities, by a lawful ordering of tasks or the placement of interruptions at task boundaries. In all cases, these types of structural changes coincide and attentionally highlight the inherent organization of the event's nontemporal information. This not only facilitates learning, but also provides a set of cognitive landmarks that can be used to reconstruct the event's total time span from beginning to end.

In sum, then, the construct of structural predictability serves to modify the traditional assumptions of the attention-based models. Although the underlying mechanisms of this approach do appear to apply to less coherent events, they do not readily explain the duration estimates of more coherent ones. To do so, it must be acknowledged that the structural predictability of an event can both influence the relative allocation of attentional resources and provide an immediate retrieval scheme for accurately remembering event durations under retrospective circumstances.

In addition to modifying the attention-based models of duration judgment, the ideas that have been presented here also refine the concept of complexity that the memory-based models are based on (Block, 1985; Ornstein, 1969). Of these two theoretical approaches, Block's cognitive-change hypothesis has been particularly successful in addressing both the direction and bias of time judgments (see Block, 1985, for a review). Again, the basic assumption of this model is that, in retrospective circumstances, people do not encode event duration into memory. Instead, an availability heuristic is used in which remembered duration is inferred from the number of nontemporal changes in memory: the intervals displaying more changes (due to event structure, cognitive processing demands, environmental context, etc.) will seem relatively longer than those containing fewer changes. Although this heuristic is once again assumed to apply to all events, regardless of their inherent structure, the results here indicate that the predicted pattern of bias is observed only for less predictable events. This, in turn, highlights the idea that it is not merely the number of changes that influence retrospective judgments. Instead, changes can play different roles in cognitive-processing activities, depending on their relative location within an event's overall context. This is best illustrated in the results of Experiments 2 and 3. In Experiment 2, there were two conditions that yielded more changes from one task to another because each was performed twice versus a third condition where each task was performed once. However, even though the number of changes was comparable in the first two conditions, it was only when task changes occurred in an unpredictable fashion that their total time spans were judged longer than those of the third group. In contrast, tasks performed twice in a predictable order were judged with a high degree of accuracy and no bias. Similar effects were observed in Experiment 3 with the placement of interruptions within versus between tasks. These sets of results demonstrate that when changes regularly recur and coincide with the hierarchical arrangement of nontemporal information, they function to decrease processing effort by drawing attention to the events' overall organization. This, in turn, allows event duration to be encoded into memory. In addition, given that changes mark nested structural relations, they also provide landmarks that enable one to accurately retrieve event duration from memory. It is only when changes conflict with the array of nontemporal information that evidence for an availability heuristic is manifested through an overestimation bias. The relative location of these changes fragment the overall organization of the event and, since duration is less likely to be encoded, people are biased by the relative number of changes in memory. In sum, then, in contrast to the traditional assumptions of the memory-based theories, an increased number of chunks and changes do not always lengthen remembered duration. Although these constructs are important ones in time-estimation behavior, one needs to consider their relative placement within the event's overall structural arrangement to predict how duration judgments will be influenced.

One final issue that merits a brief discussion is that of stimulus duration. In all three experiments reported here, 
the total duration of each to-be-judged task was always $12 \mathrm{~min}$. Although this methodological constant ensures greater comparability among the individual experiments, it may raise questions about the overall generality of results. Two points should be noted. First, variations in structural predictability at the level of event timing have been examined in a broad range of events that span many different durations. These include musical compositions 2.7 to $14.1 \mathrm{sec}$ in length (Boltz, 1992, 1995, in press), various types of ecological sounds ranging from 1.74 to $8.5 \mathrm{sec}$ (Boltz, 1992, 1994), and filmed narratives spanning 43 to 57 min (Boltz, 1995). In each case, the pattern of results revealed was identical to that observed here with manipulations of task predictability. Second, Kerr and Keil (1963) have conducted a naturalistic study on factory workers in which task ordering was varied in ways that were very analogous to those of Experiment 2. Even though these tasks varied from a few minutes to several hours in duration, the results once again converged with those reported here. Overall, then, the effects of structural predictability on remembered duration appear to be very reliable ones that generalize to a broad range of stimulus durations.

Beyond the theoretical implications of the present research, there are also some practical ones that apply to everyday behavior. One involves task performance within the work environment. Many vocations do require one to perform certain activities on a routine basis and, according to the research here, those repeatedly performed in the same order should yield shorter and more accurate judgments than those performed in a varying order. The experience of time is an important factor in the workplace because some researchers have found that task satisfaction markedly increases when time appears to transpire more quickly (Gupta \& Cummings, 1986). The reverse side of this, of course, is that an invariant task ordering across successive days may eventually induce boredom and thereby lengthen both experienced and remembered duration (Loehlin, 1959). Some insight into these issues comes from a study by Kerr and Keil (1963), who asked a group of factory workers to estimate the duration of variety versus repetitive types of jobs. In convergence with the present data, jobs routinely performed in a varying order were judged less accurately and longer than those repetitively performed in the same order. The perceived boredom of tasks did not vary across the two orders, but it was found that increased work experience (i.e., more years on the job) was correlated with greater accuracy on the repetitive types of jobs than on the variety types. In sum, then, the higher degree of predictability that emerges from task order and past learning experience both contribute to shorter and more accurate time estimates, which in turn may also influence job satisfaction, as the related study by Gupta and Cummings (1986) suggests. In situations in which task order cannot be kept constant across successive days, then, as the results of Experiment 1 indicate, simply informing workers in advance what will be required throughout a given shift should exert a similar effect on behavior.
The effects of predictability may influence a second type of behavior, both in the workplace and more generally, and it involves the calibration of temporal experience. This refers to the idea that remembered duration is often used for planning and scheduling purposes. For example, if one has several errands to run before going to work (e.g., stopping by the bank, post office, drugstore, etc.), then one must tacitly estimate in advance how long each activity will take in order to determine when to leave the house. Burt and Kemp (1994) investigated this ability by asking a group of college students to first estimate how long they thought it would take to perform different tasks (e.g., going to the bookstore to buy a stamp, consulting the card catalog in the campus library, walking from one point to another) and then comparing these estimates to the tasks' actual durations. Overall, the results revealed a high degree of variability - although the duration of some tasks were well calibrated, others were not. One factor that may be at play here but was unacknowledged in the experimental methodology is the tasks' overall degree of predictability and familiarity. Given that greater predictability results in more accurate time estimates, it would make sense that these types of tasks are the best calibrated and would therefore allow one to schedule activities in the most efficient manner. Although temporal calibration abilities have not been extensively investigated in the past literature, it is worthwhile to determine whether some of the various sources of structural coherence identified here also influence this common usage of remembered duration in everyday behavior.

\section{REFERENCES}

BERRY, W. (1976). Structural functions in music. Englewood Cliffs, NJ: Prentice-Hall.

BLock, R. A. (1982). Temporal judgments and contextual change. Journal of Experimental Psychology: Learning, Memory, \& Cognition, 8 , 530-544.

Block, R. A. (1985). Contextual coding in memory. Studies of remembered duration. In J. Michon \& J. Jackson (Eds.), Time, action, and behavior (pp. 169-178). Heidelberg: Springer-Verlag.

BLOCK, R. A. (1989). Experiencing and remembering time: Affordances, context, and cognition. In I. Levin \& D. Zakay (Eds.), Time and human cognition: A life-span perspective (pp. 333-363). Amsterdam: North-Holland.

BLOCK, R. A. (1990). Models of psychological time. In R. A. Block (Ed.), Cognitive models of psychological time (pp. 1-36). Hillsdale, NJ: Erlbaum.

BLOCK, R. A., \& REED, M. A. (1978), Remembered duration: Evidence for a contextual change hypothesis. Journal of Experimental Psychology: Human Learning \& Memory, 4, 656-665.

BLock, R. A., \& ZAKAY, D. (1997). Prospective and retrospective duration judgments: A meta-analytic review. Psychonomic Bulletin \& Review, 4, 184-197.

BoLtz, M. [G.] (1991). Some structural determinants of melody recall. Memory \& Cognition, 19, 239-251.

Boltz, M. G. (1992). The remembering of auditory event durations. Journal of Experimental Psychology: Learning, Memory. \& Cognition, 18, 938-956.

BolTZ, M. G. (1993). Time estimation and expectancies. Memory \& Cognition, 21, 853-863.

Boltz, M. G. (1994). Changes in internal tempo and effects on the learning and remembering of event durations. Journal of Experimental Psychology: Learning, Memory, \& Cognition, 20, 1154-1171. 
BoLTZ, M. G. (1995). Effects of event structure on retrospective duration judgments. Perception \& Psychophysics, 57, 1080-1096.

BoLTZ, M. G. (1996). The role of learning in time estimation behavior. Paper presented at the Meeting of the Psychonomic Society, Chicago.

BoLrz, M. G. (in press). The processing of temporal and nontemporal information in the remembering of event durations and musical structure. Journal of Experimental Psychology: Human Perception \& Performance.

Boltz, M. [G.], \& Jones, M. R. (1986). Does rule recursion make melodies easier to reproduce? If not, what does? Cognitive Psychology, 18, 389-431.

Boltz, M. [G.], Schulkind, M., \& Kantra, S. (1991). Effects of background music on the remembering of filmed events. Memory \& Cognition, 19, 593-606.

Brown, S. W. (1985). Time perception and attention: The effects of prospective versus retrospective paradigms and task demands on perceived duration. Perception \& Psychophysics, 38, 115-124.

Brown, S. W., \& STubBS, D. A. (1992). Attention and interference in prospective and retrospective timing. Perception, 21, 545-557.

BURT, C. D. B., \& KEMP, S. (1994). Construction of activity duration and time management potential. Applied Cognitive Psychology, 8, 155-168.

CONE, E. (1968). Musical form and musical performance. New York: Norton.

GOLDMAN-EISLER, F. (1972). Pauses, clauses, and sentences. Language \& Speech, 15, 103-113.

Grosjean, F., Grosjean, L., \& Lane, H. (1979). The patterns of silence: Performance structures in sentence production. Cognitive Psychology, 11, 58-81.

Gupta, S., \& Cummings, L. (1986). Perceived speed of time and task affect. Perceptual \& Motor Skills, 63, 971-980.

Hicks, R. E., Miller, G. W., \& Kinsbourne, M. (1976). Prospective and retrospective judgments of time as a function of amount of information processed. American Journal of Psychology, 89, 719-730.

JafFe, J., \& Feldstein, S. (1970). Rhythms of dialogue. New York: Academic Press.

Kahneman, D. (1973). Attention and effort. Englewood Cliffs, NJ: Prentice-Hall.

KERR, W. A., \& KEIL, R. C. (1963). A theory and factory experiment on the time-drag concept of boredom. Journal of Applied Psychology, 47, 7-9.

LERDAHL, F., \& JACKENDOFF, R. (1983). A generative theory of tonal music. Cambridge, MA: MIT Press.

LoEHLin, J. C. (1959). The influence of different activities on the perception of time. Psychological Monographs, No. 474.

MANDLER, J., \& JOHNSON, N. (1977). Remembrance of things parsed: Story structure and recall. Cognitive Psychology, 9, 111-151.
MARTIN, J. G. (1972). Rhythmic (hierarchical) versus serial structure in speech and other behavior. Psychological Review, 79, 487-509.

MilleR, G. W., Hicks, R. E., \& Willette, M. (1978). Effects of concurrent verbal rehearsal and temporal set upon judgments of temporal duration. Acta Psychologica, 42, 173-179.

Navon, D., \& Gopher, D. (1979). On the economy of the humanprocessing system. Psychological Review, 86, 214-255.

NEwTSON, D. (1973). Attribution and the unit of perception of ongoing behavior. Journal of Personality \& Social Psychology, 28, 28-38.

ORNSTEIN, R. E. (1969). On the experience of time. Baltimore, MD: Penguin.

Pierson, K. (1976). Control of walking. Scientific American, 235, 72-86. Piston, W. (1978). Harmony, New York: Norton.

PosNer, M. I., \& SNYDER, C. R. (1975). Attention and cognitive control. In R. L. Solso (Ed.), Information processing and cognition: The Loyola Symposium (pp. 55-85). Hillsdale, NJ: Erlbaum.

PoYNTER, W. D. (1983). Duration judgment and the segmentation of experience. Memory \& Cognition, 11, 77-82.

Schiffman, H. R., \& BobKo, D. J. (1974). Effects of stimulus complexity on the perception of brief temporal intervals. Journal of Experimental Psychology, 103, 156-159.

Thomas, E. A. C., \& CANTOR, N. E. (1978). Interdependence between the processing of temporal and nontemporal information. In J. Requin (Ed.), Attention and performance VII (pp. 43-62). Hillsdale, NJ: Erlbaum.

THORNDYKE, P. (1977). Cognitive structures in comprehension and memory of narrative discourse. Cognitive Psychology, 9, 77-110.

ToDd, N. (1985). A model of expressive timing in tonal music. Music perception, 3, 33-58.

ZAKAY, D. (1989). Subjective time and attentional resource allocation: An integrated model of time estimation. In I. Levin \& D. Zakay (Eds.), Time and human cognition: A life span perspective (pp. 365397). Amsterdam: North-Holland.

ZAKAY, D. (1990). The evasive art of subjective time measurement: Some methodological dilemmas. In R. A. Block (Ed.), Cognitive models of psychological time (pp. 59-84). Hillsdale, NJ: Erlbaum.

ZAKAY, D. (1993). Relative and absolute duration judgments under prospective and retrospective paradigms. Perception \& Psychophysics, $54,656-664$

ZAKAY, D., \& MERAN, V. (1988). A meta-analysis of time estimation research. Unpublished manuscript, Tel-Aviv University.

ZaKay, D., Tsal, Y., Moses, M., \& Shahar, I. (1994). The role of segmentation in prospective and retrospective time estimation processes. Memory \& Cognition, 22, 344-351.

(Manuscript received December 9, 1996; revision accepted for publication June 14,1997 .) 